\title{
Fermented jackfruit leaf beverage offers new affordable and effective diabetes therapy
}

\author{
${ }^{1 *}$ Koh, S.P., ${ }^{1}$ Maarof, S., ${ }^{2}$ Sew, Y.S., ${ }^{1}$ Sabidi, S., ${ }^{1}$ Abdullah, R, ${ }^{1}$ Mohd Danial, A., ${ }^{1}$ Nur Diyana, A. \\ and ${ }^{3}$ Mustaffa, $R$ \\ ${ }^{1}$ Food Science and Technology Research Centre, Malaysian Agricultural Research and Development \\ Institute (MARDI), Persiaran MARDI-UPM, 43400 UPM Serdang, Selangor, Malaysia. \\ ${ }^{2}$ Biotechnology and Nanotechnology Research Centre, Malaysian Agricultural Research and Development \\ Institute (MARDI), Persiaran MARDI-UPM, 43400 UPM Serdang, Selangor, Malaysia. \\ ${ }^{3}$ Horticulture Research Centre, Malaysian Agricultural Research and Development Institute (MARDI), \\ Persiaran MARDI-UPM, 43400 UPM Serdang, Selangor, Malaysia.
}

\begin{abstract}
Article history:
Received: 11 April 2020

Received in revised form: 17

May 2020

Accepted: 24 May 2020

Available Online: 27

December 2020

Keywords:

Fermentation,

Jackfruit leaf,

Anti-diabetic effect,

Streptozotocin-induced,

Haematology profile
\end{abstract}

\section{DOI:}

https://doi.org/10.26656/fr.2017.4(S6).012

\begin{abstract}
Diabetes mellitus is complicated multifactorial disorders characterised by hyperglycemia or glucose intolerance. Globally, the number of people suffering from diabetes has increased steadily. Jackfruit leaf, a rich source of phytochemicals offers the opportunity for the development of value-added products. A fermented jackfruit leaf beverage product was developed using symbiotic culture of bacteria and yeast (SCOBY) as cost-effective beneficial treatment for mankind in the management of diabetes. The effectiveness of this fermented jackfruit leaf beverage as an anti-diabetic therapy agent was evaluated using Streptozotocin-induced Sprague Dawley rats model for 4 weeks. The findings depict that, there was a remarkable reduction of blood glucose level in fermented jackfruit leaf-treated diabetic rats as opposed to non-treated diabetic rats, comparable with metformin-treated diabetic rats. The body weight and various relative organ weights of diabetic rats treated with fermented jackfruit leaf beverage showed a healthy recovery sign from diabetes symptoms. The blood haematology profile, liver and kidney function analysis revealed no toxic effect of prolong fermented jackfruit leaf beverage consumption. These results indicated that fermented jackfruit leaf beverage has similar anti-diabetic properties with commercial anti-diabetic drugs with no adverse side effect. Promisingly, it offers a cheaper source to alleviate the diabetes epidemic in a natural way.
\end{abstract}

\section{Introduction}

Diabetes, a silent killer is the most common noncommunicable disease, characterized by hyperglycemia due to the presence of insufficient Insulin or peripheral resistance to insulin action in your body. The insulin is a hormone produced in the pancreas which plays a critical role to enable the glucose to be transported into the cells for growth and energy. In diabetes people, either the pancreas is damaged (Type 1, $10 \%$ ) or insulin resistance (Type $2, \sim 90 \%$ ), they will have a problem to transport glucose into their cells and experienced hyperglycemia effect which resulting some health complications associated with it, including constant tiredness, excessive thirst, slow healing of cuts and wounds and etc. Serious diabetes complications have been linked directly with heart attack and stroke, high blood pressure, kidney failure, nerve damage, poor blood circulation, damage to eye, etc. (Peppa and Vlassara, 2005; Contreras and Novakofski, 2010).

In Malaysia, it was reported that approximately 3.6 million populace are suffering from diabetes which is one of the highest in the world. A diabetes prevalence rate of $31.3 \%$ for adults (aged above 18) was reported and forecast about 7 million Malaysian will be affected by 2025 (Bernama, 2019). Globally, the diabetes epidemic is increased exponentially, mainly because of an increase in the prevalence of obesity population worldwide, contributing to the rising of prevalence Type 2 diabetes mellitus. Thus, it surges huge demands for the diabetes drug market. Global diabetes therapeutics market was valued to reach $\$ 186,842$ million by 2023 with compound annual growth rate (CAGR) of $16.0 \%$ by 2023 (Allied Market Research, 2019). The impact of diabetes on society is substantial as it can impose a major 
economic burden on the healthcare system and the national economy. In the current market, few therapeutic drugs have been introduced to regulate blood glucose metabolism to a normal state. The cost of treating diabetes and also its complications is unpredictable high. Therefore, the development of affordable and effective diabetes therapy with less adverse side effect is a challenge and highly demanded.

In recent year, plant medicine became popular in diabetic control as it is considered to be less toxic with lower side effect than synthetic drugs (Wang et al., 2016; Tafesse et al., 2017). Fermentation of selected plant foods also reported have disease-preventive properties with the presence of functional phytochemicals which contributes to antioxidant properties (Yeo and Ewe, 2015). Jackfruit (Artocarpus heterophyllus L.), one of the profitable multipurpose food crops has been recognized with its multiple functional components related to human health. Extracts from various part of jackfruit plant have been applied as traditional medical treatment as it possesses antioxidant, wound healing effect, anti-inflammatory, anti-bacterial, etc (Baliga et al., 2011). Most of the pharmacological properties are contributed by the presence of phytonutrients including flavonoids, stilbenoids, aryl benzofurans and prenylflavones (Jagtap and Bapat, 2010; Swami et al., 2012). Jackfruit pulp extract was reported capable to inhibit the haemoglobin glycation, thus possess interesting anti-diabetic activity. It was reported that antioxidant and radical scavengers can prevent glycation end products formation (Biworo et al., 2015).

Numerous studies have shown the jackfruit leaf has pharmacological properties. Jackfruit leaf provides valuable fodder for cattle and others livestock with the presence of phytochemicals like flavonoids, tannins, saponins and carbohydrates which was reported promote the growth performance of kids grazing (Das and Ghosh, 2007). Extracts of jackfruit leaf showed no toxicity effect at the dose study of $2000 \mathrm{mg} / \mathrm{kg}$ (Bhattacharjee and Dutt, 2013). Therefore, there is no toxicity concern about the application use jackfruit leaf as a functional beverage. Furthermore, the extract of jackfruit leaf was demonstrated to have potential antioxidant and antibacterial properties (Loizzo et al., 2010). Nevertheless, the taste of jackfruit leaf is awful and not suitable for direct consumption. With the potential of antioxidant activity and abundant availability of jackfruit leaf underutilized, we have developed a palatable fermented jackfruit leaf beverage via microbiological action to produce more secondary metabolites which may contribute to anti-diabetic properties and improve its organoleptic acceptability for ready-to-drink. In this study, Streptozotocin-induced Sprague Dawley rats model were designed to examine the anti-diabetic effect of fermented jackfruit leaf beverage for a duration period of 4 weeks treatment. Metformin, commercial antidiabetic drugs were used as a positive control for comparison purpose. The blood biochemistry and histopathology organs of all rats group were investigated also to examine its safety concern for prolong consumption.

\section{Materials and methods}

\subsection{Jackfruit leaf powder}

The mature leaves of jackfruit tree (Artocarpus heterophyllus L, Tekam Yellow variety) were collected from Agriculture Park in Lanchang, Pahang. The leaves were thoroughly washed clean and dried in the oven at $50^{\circ} \mathrm{C}$ for $24 \mathrm{hrs}$ to achieve a moisture content of $8-10 \%$ before grind into powder using an ultra centrifugal mill (Retsch, model: ZM200). The dried powder was packed in a laminated aluminium bag and stored in chill condition $\left(4 \pm 1^{\circ} \mathrm{C}\right)$ to maintain the freshness of leaf powder for further use.

\subsection{Preparation of fermented jackfruit leaf beverage}

Jackfruit leaf was used as a substrate for the production of fermented jackfruit leaf (JL) beverage product. The jackfruit leaf suspension with the concentration of $5 \%(\mathrm{w} / \mathrm{v})$ was prepared and sterilized it by autoclaving before inoculated with mixed pure cultures consists of: a) yeast (Dekkera sp.) and b) acetic acid bacteria (Komagataiebacter sp.). These two starter cultures were selected from MARDI's Collection of Functional Food Cultures. Following a week of the fermentation process, the supernatant was collected after centrifuged at $10,000 \mathrm{rpm}$ for 10 mins to remove the remaining substrate residue. The collected supernatant was pasteurised at $90^{\circ} \mathrm{C}$ for 30 mins to stop the fermentation process and then stored in chilled condition for further study.

\subsection{Animal care}

The experimental animal protocol was conducted according to the guidelines and was approved by the Animal Ethics Committee of MARDI (20180810/R/ MAEC00025). Male Sprague-Dawley rats (8 weeks old) were placed in the Animal Metabolism, Toxicology and Reproductive Centre (AMTREC MARDI, Serdang). The rats were maintained under standard conditions: room temperature $\left(25 \pm 2^{\circ} \mathrm{C}\right)$, relative room humidity $(55 \%)$ and alternating light-dark cycle $(12 \mathrm{hr} / 12 \mathrm{hr})$ with access to standard rat pellets and water ad libitum for the entire experimental period. All animals were acclimatised for two weeks in the cage before proceeding to anti-diabetic study. 


\subsection{Streptozotocin-induced diabetic rat study}

To induce diabetes mellitus in the experimental rat model, male Sprague-Dawley rats were treated via intraperitoneal injection with Streptozotocin (STZ, 40 $\mathrm{mg} / \mathrm{kg}$ ) freshly prepared in a cold $0.1 \mathrm{M}$ sodium citrate buffer ( $\mathrm{pH} 4.5$ ) prior to fasting for approximately $16 \mathrm{hrs}$ with only water provided ad libitum. The rats in the normal group were injected intraperitoneal with sodium citrate buffer ( $\mathrm{pH} 4.5$ ) as control (CTRL N, $\mathrm{n}=6$ ). Three days after induction, a drop of blood from the tail vein of each rat was obtained using a scissor. The blood was analysed using a commercial glucometer (ACCU-CHECK Active Glucose Monitor, Roche, Germany) to determine hyperglycemia development. The fasting rats with a blood glucose level $>11 \mathrm{mmol} / \mathrm{L}$ after injection were considered diabetic. The successfully established type 2 diabetic rats were divided into 3 group: a) negative control (CTRL -) group without treatment; b) positive control $(\mathrm{CTRL}+)$ group treated with Metformin $(4.5 \mathrm{mg} / \mathrm{kg})$; c) fermented jackfruit leaf (JL) group treated with fermented JL beverage $(1.5 \mathrm{~mL} / \mathrm{kg})$. All treated diabetic rats were administered orally by oral gavage technique for 28 consecutive days. Bodyweight and blood glucose of each rat was recorded at the initiation treatment and once weekly until the day of necropsy. On day 29, rats were sacrificed in the $\mathrm{CO}_{2}$ chamber and blood was drawn afterwards via the brachial artery and stored in tubes containing anticoagulant EDTA-2K. Red blood cell, platelets, white blood cell, haemoglobin and hematocrit were obtained by running automated haematology analyzer (Exigo Blood Haematology Analyzer, Sweden). Serum samples were obtained by centrifuging blood samples (Centrifuge S417R, Eppendorf, CA, USA) at 4,000 rpm for $10 \mathrm{mins}$ under the controlled temperature of $4^{\circ} \mathrm{C}$. Then, serums samples were analysed using a clinical chemistry autoanalyzer (DIRUI CS-300, China) to identify alanine aminotransferase (ALT), alkaline phosphatase (ALP), aspartate aminotransferase (AST), total protein, creatinine, urea readings. The data obtained will be compared with the normal range of Sprague-Dawley rats as reported by Petterino and Argentino-Storino (2006).

\subsection{Histopathology examination}

The sacrificed rats were then dissected and the organs (stomach, liver, heart, kidney and spleen) were harvested. All organs were fixed in neutral buffered $10 \%$ formalin solution and were embedded in paraffin, sectioned, stained with haematoxylin and eosin (H\&E) before examined individual organ tissue under microscope (Leica, Germany).

\subsection{Statistical analysis}

All data were performed using one-way analysis of variance (ANOVA) based on Duncan's test with the statistical significance set at the level of $P<0.05$. Results are presented as mean \pm standard deviation of replicated samples. All analyses were conducted using statistical analysis software, IBM SPSS Statistic 22.0 (IMB Corp., USA).

\section{Results and Discussion}
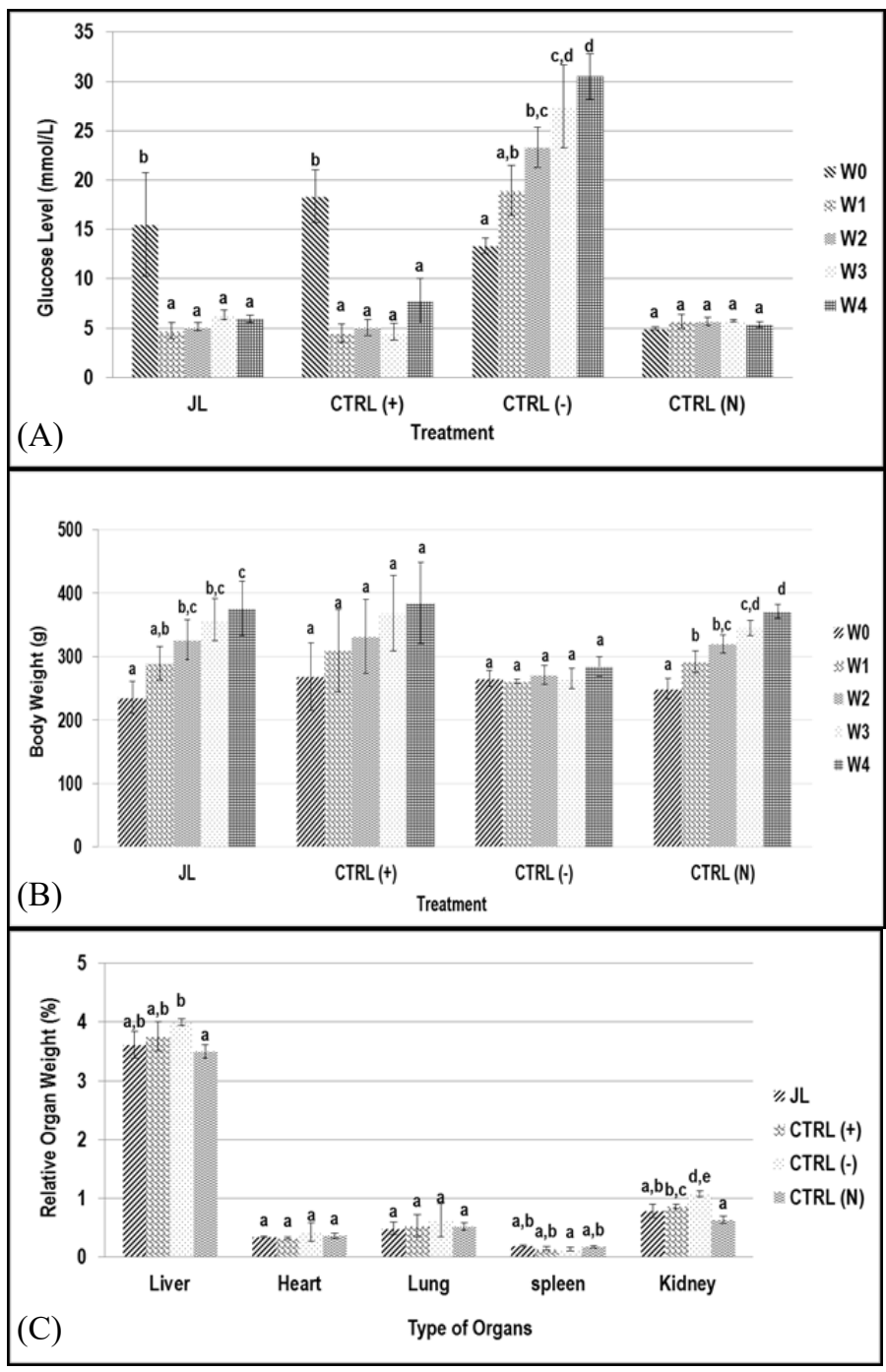

Figure 1. Comparative study of glucose level, body weight and relative organ between treated diabetic induced and control rats group. The results of male Sprague-Dawley rats $(n=6)$ are expressed as mean \pm standard deviation. The differences between the control and treated rats group were determined by one way ANOVA $(P<0.05)$. JL - fermented jackfruit leaf-treated diabetic rats; CTRL $(+)$ : Metformintreated diabetic rats; CTRL (-): Diabetic rats; CTRL (N): normal control rats.

Fermented jackfruit leaf beverage was produced using selected SCOBY strain to improve bioactive metabolites and its astringent taste (Koh et al., 2018) with the aim to value-added the abundant of 
underutilized jackfruit leaf which was known to have pharmacological properties. In this study, Streptozotocin (STZ), a naturally occurring alkylating antineoplastic agent was used to induce Type 2 Diabetic rats as it is preferentially toxic to the insulin-producing pancreatic beta cells, resulting in hyperglycemia. A single dose of $40 \mathrm{mg} / \mathrm{kg} \mathrm{STZ}$ effectively induced diabetes on rats by day 3 after STZ injection with the glucose level reading more than $11 \mathrm{mmol} / \mathrm{L}$. The STZ -induced diabetic rats were divided into 3 groups and observed for another 2 days to ensure the blood glucose level of selected rats remained in the diabetic state before proceeding to diabetic treatment. Generally, the time to recovery from diabetic state to normal blood glucose level of all diabetic rats group for different treatment was significantly different $(P<0.05)$ as shown in Figure 1a. Both fermented JL- and Metformin-treated diabetic rats group showed a sign of fast recovery after one week orally administration with fermented JL beverage and Metformin, respectively. On the contrary, the untreated diabetic rat group (CTRL -) showed a steady increment of blood glucose level at the interval of one week and reached a severe diabetic state at week 4 . In addition, it was observed that rats remained in the diabetic state for long period time has resulting poor body condition, whereby no significant difference $(P<0.05)$ of rats body weight gain as opposed week 0 (Figure 1b).

Surprisingly, the dose of $1.5 \mathrm{~mL}$ fermented JL beverage on daily administration on diabetic rats showed a promising reduction of glucose level within one week treatment period and confirmed its capability to recover from the diabetic state. The fermented JL-treated diabetic rat body weight gain increased gradually to a healthier state after one-month treatment as compared with diabetic rats group. The finding has supported the antidiabetic property of fermented JL beverage and implied the comparative effectiveness to control diabetic disease when compared with the commercial anti-diabetic drug, Metformin. Previously, this fermented jackfruit leaf beverage has been reported to have significant improvement of 2,2-Diphenyl-1-Picrlhydrayl (DPPH) free radical scavenging activities than jackfruit leaf without fermentation (Aziz et al., 2018) with the presence of health-promoting phytochemicals (e.g. catechin, chlorogenic acid, caffeic acid, quinic acid, quercetin). These flavonoids have been studied earlier and denote a protective role to regulate blood glucose (Ong et al., 2013, Oboh et al., 2015, Eid and Hadad, 2017). Particularly, chlorogenic acid was presented capable to inhibit both $\alpha$-amylase and $\alpha$-glucosidase (key enzymes linked to type 2 diabetes) and improve glucose and lipid metabolism via the activation of AMPdependent kinase (Ong et al., 2013; Oboh et al., 2015). Numerous studies confirmed that both antioxidant and antiglycation properties of flavonoid compounds (e.g. protocatechuic acid, catechin, chlorogenic acid, gallic acid) have therapeutic potential in treating diabetic complications (Omar et al., 2011; Yagi et al., 2013; Biworo et al., 2015). Furthermore, different solvent extracts of jackfruit leaf with a substantial amount of flavonoid content also proven exert hypoglycaemic effect in STZ-diabetic rats which might be supported by the strong scavenge diphenylpicrylhydrazyl radical and chelating effect of ferrous iron activities (Omar et al., 2011). An additional study by Jagtap et al. (2011) revealed an improved antioxidant capacity of fermented jackfruit product which exert a protective role against radiation-induced DNA damage. Taken together, the excellent performance of fermented JL beverage in reducing blood glucose of diabetic rats to the normal state is mainly contributing by the presence of health functional flavonoids which implies a protective role to control diabetic complications with no side effect.

Generally, all relative organs weight of all treated diabetic rats group showed minor difference with negative control and normal control rat group (Figure 1c). Nevertheless, the histopathology analysis revealed no inflammatory symptom observed in all rats organ tissues of liver, heart, lung, spleen and kidney as depicted in Figure 2. The haematology analysis was conducted on all blood samples to monitor the concentration of red blood cells, white blood cells, platelets, haemoglobin and \% hematocrit. On the whole, all rats group showed the acceptable normal range of haematology profile except for diabetic rats without treatment (CTRL -) showed slightly lower white blood cell concentration (Table 1).

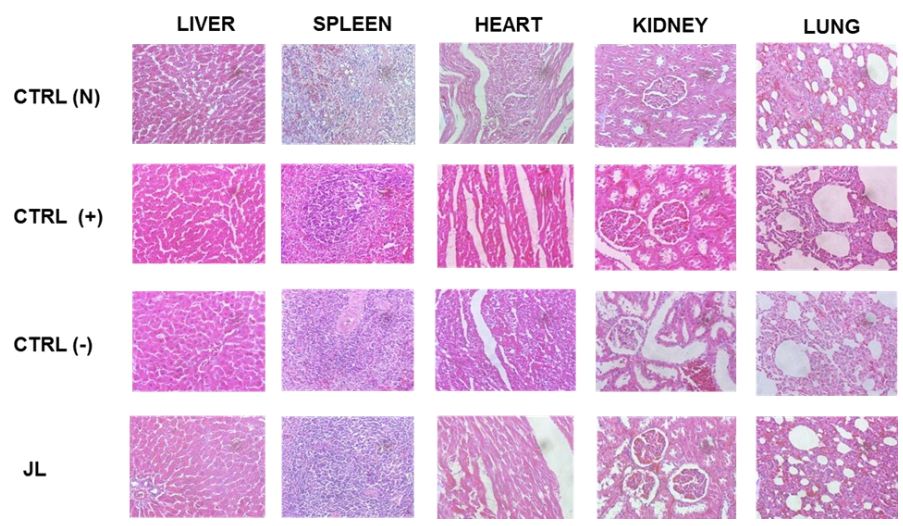

Figure 2. Histopathology profile of treated diabetic induced and control rats group of selected organs (liver, spleen, heart, kidney, lung). JL - fermented jackfruit leaf-treated diabetic rats; CTRL (+): Metformin-treated diabetic rats; CTRL (-): Diabetic rats; CTRL (N): normal control rats.

The spectrum of serum biochemistry was analysed to evaluate any abnormal of liver enzymes, urea and creatinine level. The most important indicators in assessing liver injury are the alanine aminotransferase, 
Table 1. Haematology analysis and serum biochemistry profile between treated diabetic-induced and control rats group

\begin{tabular}{|c|c|c|c|c|c|c|c|c|c|c|c|c|c|}
\hline \multirow[b]{2}{*}{ Treatment } & \multicolumn{5}{|c|}{ Haematology profile } & \multicolumn{6}{|c|}{ Liver Function Profile } & \multicolumn{2}{|c|}{ Kidney Function Profile } \\
\hline & $\begin{array}{l}\text { Red blood cell } \\
\quad\left(10^{12} /\right)\end{array}$ & $\begin{array}{l}\text { White blood cell } \\
\left(10^{9} / 1\right)\end{array}$ & $\begin{array}{l}\text { Platelet } \\
\left(10^{9} /\right)\end{array}$ & $\begin{array}{l}\text { Hemoglobin } \\
\text { (g/dl) }\end{array}$ & $\begin{array}{l}\text { Hematocrit } \\
(\%)\end{array}$ & $\begin{array}{c}\text { Alanine } \\
\text { aminotransferase } \\
\text { (U/I) }\end{array}$ & $\begin{array}{c}\text { Aspartate } \\
\text { aminotransferase } \\
\text { (UII) }\end{array}$ & $\begin{array}{l}\text { Alkaline } \\
\text { phosphatase } \\
\text { (U/I) }\end{array}$ & $\begin{array}{l}\text { Total protein } \\
(\mathrm{g} / \mathrm{l})\end{array}$ & $\begin{array}{l}\text { Albumin (A) } \\
(\mathrm{g} / \mathrm{l})\end{array}$ & $\begin{array}{c}\text { Globulin (G) } \\
(\mathrm{g} / \mathrm{l})\end{array}$ & $\begin{array}{c}\text { Urea } \\
\text { mmol/I) }\end{array}$ & $\begin{array}{c}\text { Creatinine } \\
(\mu \mathrm{mol} / \mathrm{I})\end{array}$ \\
\hline $\operatorname{CTRL}(+)$ & $10.62 \pm 0.57^{\mathrm{a}}$ & $8.30 \pm 1.17^{\mathrm{ab}}$ & $959.00 \pm 203.66^{\mathrm{a}}$ & $19.57 \pm 0.80^{3}$ & $55.97 \pm 3.39^{\mathrm{a}}$ & $142.29 \pm 110.38 \mathrm{~cd}$ & $226.00 \pm 156.47^{\mathrm{b}}$ & $224.33 \pm 34.31^{\mathrm{b}}$ & $64.23 \pm 3.25 \mathrm{ab}$ & $36.36 \pm 1.69 \mathrm{a}$ & $27.90 \pm 1.67$ abc & $7.72 \pm 1.02^{b}$ & $22.33 \pm 4.51^{\circ}$ \\
\hline CTRL (-) & $10.70 \pm 1.09^{\mathrm{a}}$ & $7.18 \pm 3.93^{b}$ & $498.25 \pm 217.07 \mathrm{a}$ & $20.30 \pm 1.96^{\mathrm{a}}$ & $60.53 \pm 6.85^{\mathrm{a}}$ & $296.33 \pm 130.30$ bo & $745.00 \pm 0.00=$ & $488.33 \pm 134.88 \mathrm{a}$ & $54.58 \pm 3.63^{\circ}$ & $31.88 \pm 1.72^{b}$ & $22.70 \pm 3.67^{\circ}$ & $22.02 \pm 4.34^{\text {a }}$ & $24.50 \pm 9.75^{\mathrm{a}}$ \\
\hline CTRL (N) & $10.91 \pm 1.64^{a}$ & $13.48 \pm 3.13^{\mathrm{a}}$ & $1175.75 \pm 402.50^{\mathrm{a}}$ & $21.13 \pm 3.02^{\mathrm{a}}$ & $60.95 \pm 9.34^{a}$ & $43.67 \pm 3.10^{d}$ & $90.33 \pm 12.34 \mathrm{~b}$ & $169.75 \pm 27.94$ b & $68.80 \pm 3.45^{a}$ & $39.70 \pm 1.40^{\mathrm{a}}$ & $29.10 \pm 2.30 \mathrm{ab}$ & $5.97 \pm 0.79 b$ & $24.25 \pm 2.22 \mathrm{a}$ \\
\hline $\mathrm{JL}$ & $10.03 \pm 0.82^{\mathrm{a}}$ & $9.20 \pm 3.58^{\mathrm{ab}}$ & $1029.00 \pm 467.74^{a}$ & $19.13 \pm 1.31^{\mathrm{a}}$ & $55.20 \pm 3.90^{\mathrm{a}}$ & $50.33 \pm 10.41^{\mathrm{d}}$ & $101.33 \pm 27.80^{b}$ & $202.00 \pm 28.93^{b}$ & $65.77 \pm 3.40^{\mathrm{a}}$ & $36.66 \pm 2.81^{a}$ & $29.10 \pm 1.21 \mathrm{ab}$ & $6.74 \pm 2.09 \mathrm{~b}$ & $22.33 \pm 0.58$ a \\
\hline $\begin{array}{c}\text { Normal } \\
\text { range }\end{array}$ & $7.10-10.20$ & $8.10-19.00$ & $370.00-1383.00$ & $13.40-18.40$ & $37.90-49.90$ & $13.50-64.00$ & $29.50-172.20$ & $90.50 \cdot 324.30$ & 57.00- 87.00 & $32.00-44.00$ & $30.00-43.00$ & $6.90-31.70$ & $26.50 \cdot 70.70$ \\
\hline
\end{tabular}

The results of male Sprague-Dawley rats $(n=6)$ are expressed as mean \pm standard deviation. The differences between the control and treated rats group were determined by one way ANOVA $(P<0.05)$. JL - fermented jackfruit leaf-treated diabetic rats; CTRL $(+)$ : Metformin-treated diabetic rats; CTRL (-): Diabetic rats; CTRL (N): normal control rats.

aspartate aminotransferase and alkaline phosphate. The liver function profile of diabetic rats without treatment showed a drastic increment of alanine aminotransferase, aspartate aminotransferase and alkaline phosphate in blood serum indicating liver injury symptoms in accordance with the number of liver abnormalities occur related with diabetes mellitus complications as reported in the past (Mohamed et al., 2016). Liver damage is a serious complications among diabetic patients. Therefore a safe and effective medication is critical in the management of diabetes. Alternatively, both metformin and fermented JL treated diabetic rats showed good promising health recovery sign as their liver function remained within a normal healthy range (Table 1). Even though the kidney function profile of all rats was within a normal range, but it was noticed that diabetic rats showed slightly higher urea concentration in contrast to treated diabetic and normal control rats group (Table 1). In diabetes condition, the blood vessels in the kidneys may injure and not able to clean the blood properly, subsequently, the blood urea nitrogen levels will rise. If this condition is not under control, it may cause kidney failure and the resulting loss of appetite, fatigue and weakness as observed in diabetic rats without treatment and physical observation has shown no significant difference $(P>0.05)$ in body weight gain within 4 weeks. Overall, diabetic rats treated with fermented JL beverage or Metformin demonstrated effective anti-diabetic therapy. Allied with the concept food as medicine, fermented JL beverage offers a great opportunity to new anti-diabetic therapy for controlling the blood glucose in a natural way through daily consumption with no side effect as confirmed by the evidence findings.

\section{Conclusion}

The treatment of diabetes mellitus is a challenging task, particularly in exploring a safe and effective medication to tackle the diabetes epidemic with a less adverse side effect. The fermented JL beverage has been confirmed to have anti-diabetic effect as supported by the evidence shown in Streptozotocin-induced diabetic rats model. By transforming jackfruit leaves into palatable fermented jackfruit leaf beverage via controlled submerged fermentation using SCOBY pure cultures, it offers numerous opportunities for diabetes therapeutic market with less cost investment involved. Value-added fermented jackfruit leaf beverage provides new insights and open a great opportunity to maximize the use of abundant agricultural waste to develop new downstream processing and generate new income revenue. Future works will focus on the pre-clinic trial on diabetic patients.

\section{Acknowledgments}

This work was conducted with the support from Horticulture Research Centre, Malaysian Agricultural Research and Development Institute (MARDI) and financially funded by Malaysia Development Fund RMK 11 (P21003004050001).

\section{References}

Allied Market Research (2019). Diabetes Therapeutics Market. Retrieved on January 6, 2019 from Allied Market Research website: www.alliedmarketresearch.com/diabetestherapeutics-market

Aziz, N., Koh, S.P., Abdullah, R., Abdul Hamid, N.S. and Mustaffa, R. (2018). The Phytochemical and antioxidant characteristics of fermented jackfruit (Artocarpus heterophyllus L.) leaves using single and mixed starter culture, Journal of Food Science and Engineering, 8, 55-60. https:// doi.org/10.17265/2159-5828/2018.01.006

Baliga, M.S., Shivashanjara, A.R., Haniadka, R., Dsouza, J. and Bhat, H.P. (2011). Phytochemistry, nutritional and pharmacological properties of 
Artocarpus heterophyllus Lam (jackfruit): a review. Food Research International, 44(7), 1800-1811. https://doi.org/10.1016/j.foodres.2011.02.035

Bernama (2019). Close to 1 in 3 adults diabetic by 2025, says health minister. Retrieved on March 26, 2019 from New Strait Times website: www.nst.com.my/ news/nation/2019/03/473136/close-1-3-adultsdiabetic-2025-says-health-minister

Bhattacharjee, C. and Dutt, A. (2013). Phytochemical and acute toxicity study leave of Artocarpus heterophyllus Lam. International Journal of Allied Medical Sciences and Clinical Research (IJAMSCR), 1(2), 78-81.

Biworo, A., Tanjung, E. Iskandar, Khairina. and Suhartono, E. (2015). Antidiabetic and antioxidant activity of jackfruit (Artocarpus heterophyllus) extract. Journal of Medical and Bioengineering, 4, 318-323. https://doi.org/10.12720/jomb.4.4.318-323

Contreras, C.L. and Novakofski, K.C. (2010). Dietary advanced glycation end products and aging. Nutrients, 2(2), 1247-1265. https://doi.org/10.3390/ nu2121247

Das, A. and Ghosh, S.K. (2007). Effect of partial concentrates with jackfruit (Artocarpus heterophyllus) leaves on growth performance of kids grazing on native pasture of Tripura, India. Small Ruminant Research, 67(1), 36-44. https:// doi.org/10.1016/j.smallrumres.2005.09.019

Eid, H.M. and Haddad, P.S. (2017). The Antidiabetic Potential of Quercetin: Underlying Mechanisms. Current Medical Chemistry, 24(4), 355-364. https:// doi.org/10.2174/0929867323666160909153707

Jagtap, U.B. and Bapat, V.A. (2010). Artocarpus of its traditional uses, phytochemistry and pharmacology. Journal of Ethnopharmacology, 129(2), 142-166. https://doi.org/10.1016/j.jep.2010.03.031

Jagtap, U.B., Waghware, S.R., Lokhande, V.H., Suprasanna, P. and Bapat, V.A. (2011). Preparation and evaluation and antioxidant capacity of Jackfruit (Artocarpus heterophyllus Lam.) wine and its protective role against radiation induced DNA damage. Industrial Crops and Products, 34(3), 15951601. https://doi.org/10.1016/j.indcrop.2011.05.025

Koh, S.P., Nur Diyana, A., Aziz, N., Hamid, N.S.A., Abdullah, R. and Mustaffa, R. (2018). The role of SCOBY strains improving functional bioactivities of fermented jackfruit beverages, presented at Asian Federation of Biotechnology Malaysia Chapter International Symposium 2018 (AFOBMCIS 2018) Roles of Biotechnology in Sustainable Growth and Development. Sarawak: AFOB Malaysia Chapter.

Loizzo, M.R., Tundis, R., Chandrika, U.G., Abeysekera,
A.M., Menichini, F. and Frega, N.G. (2010). Antioxidant and antibacterial activities on foodborne pathogens of Artocarpus heteroplyllus Lam. (Moraceae) leaves extracts. Journal of Food Science, 75(5), M291-M295. https://doi.org/10.1111/j.17503841.2010.01614.X

Mohamed, J., Nazratun Nafizah, A.N., Zariyantey, A.H. and Budin, S.B. (2016). Mechanisms of diabetesinduced liver damage - The role oxidative stress and inflammation. Sultan Qaboos University Medicine Journal, 16(2), 132-141. https://doi.org/10.18295/ squmj.2016.16.02.002

Oboh, G., Agunloye, O.M., Adefegha, S.A., Akinyemi, A.J. and Ademiluyi, A.O. (2015). Caffeic and chlorogenic acids inhibit key enzymes linked to type 2 diabetes (in vitro): a comparative study. Journal of Basic and Clinical Physiology and Pharmacology, 26(2), 165-70. https://doi.org/10.1515/jbcpp-20130141

Omar, H.S., El-Beshbishy, H.A., Moussa, Z., Taha, K.F. and Singab, A.N.B. (2011). Antioxidant activity of Artocarpus heterophyllus Lam. (JackFruit) leaf extracts: remarkable attenuation of hyperglycemia and hyperlipidemia in Streptozocin-diabetic rats. The Scientific World Journal, 11, 860970. https:// doi.org/10.1100/tsw.2011.71

Ong, K.W., Hsu, A. and Tan, B.K.H. (2013). Antidiabetic and anti-lipidemic effects of chlorogenic acid are mediated by AMPK activation. Biochemical Pharmacology, 85(9), 1341-1351. https:// doi.org/10.1016/j.bcp.2013.02.008

Peppa, M. and Vlassara, H. (2005). Advanced glycation end products and diabetic complications: a general overview. Hormones, 4(1), 28-37. https:// doi.org/10.14310/horm.2002.11140

Petterino, C. and Argentino-Storino, A. (2006). Clinical chemistry and haematology historical data in control Sprague-Dawley rats from pre-clinical toxicity studies. Experimental and Toxicologic Pathology, 57 (3), 213-219. https://doi.org/10.1016/ j.etp. 2005.10 .002

Swami, S.B., Thakor, N.J., Haldankar, P.M. and Kalse, S.B. (2012). Jackfruit and its many functional components as related to human health: a review. Comprehensive reviews in Food Science and Food Safety, 11(6), 565-576. https://doi.org/10.1111/ j.1541-4337.2012.00210.x

Tafesse, T.B., Hymate, A., Mekonen, Y. and Tadesse, M. (2017). Antidiabetic activity and phytochemical screening of extracts of the leaves of Ajuga remota Benth on alloxan-induced diabetic mice. BMC Complementary and Alternative Medicine. https:// doi.org/10.1186/s12906-017-1757-5 
Wang, J., Teng, L., Liu, Y., Hu, W., Chen, W., Hu, X., Wang, Y. and Wang D. (2016). Studies on the antidiabetic and antinephritic activities of Paecilomyces hepiali water extract in dietstreptozocin-induced diabetic Sprague Dawley rats. Journal of Diabetes Research, 2016, 4368380. https://doi.org/10.1186/s12906-017-1757-5

Yagi, S., Drouart, N., Bourgaud, F., Henry, M., Chapleur, Y. and Laurain-Matter, D. (2013). Antioxidant and antiglycation properties of hydnora johannis roots. South Africa Journal of Botany, 84, 124-127. https://doi.org/10.1016/j.sajb.2012.10.006

Yeo, S.K. and Ewe, J.A. (2015). Effect of fermentation on the phytochemical contents and antioxidant properties of plant foods. In Holzapfel, W. (Ed.) Advances in Fermented Foods and Beverages, p. 107 -122. USA: Woodhead Publishing. https:// doi.org/10.1016/B978-1-78242-015-6.00005-0 